Association for Information Systems AIS Electronic Library (AISeL)

Wirtschaftsinformatik Proceedings 1999

Wirtschaftsinformatik

February 1999

\title{
Cuparla - Telekooperation im Stuttgarter Kommunalparlament
}

Gerhard Schwabe

Universität Koblenz-Landau, schwabe@uni-koblenz.de

Helmut Krcmar

Universität Hohenheim, krcmar@uni-hohenheim.de

Follow this and additional works at: http://aisel.aisnet.org/wi1999

\section{Recommended Citation}

Schwabe, Gerhard and Krcmar, Helmut, "Cuparla - Telekooperation im Stuttgarter Kommunalparlament" (1999). Wirtschaftsinformatik Proceedings 1999. 32.

http://aisel.aisnet.org/wi1999/32 


\section{Cuparla - Telekooperation im Stuttgarter Kommunalparlament}

Gerhard Schwabe

Universität Koblenz-Landau (schwabe@uni-koblenz.de)

Helmut Krcmar

Universität Hohenheim ( $\mathrm{krcmar@uni-hohenheim.de)}$

\section{Inhalt}

1 Einleitung

2 Ziele des Projekts CUPARLA

3 Gemeinderatsarbeit

3.1 Dauer der Gemeinderatsarbeit

3.2 Kooperationspartner der Gemeinderatsarbeit

3.3 Orte der Gemeinderatsarbeit

4 Die Cuparla-Software

4.1 Prozeßorientierter Ansatz

4.2 Materialorientierter Ansatz

4.3 Kontextorientierter Ansatz

4.4 Umsetzung

4.5 Weitere Produkte

5 Erfahrungen und Wirkungen

5.1 Akzeptanz der Technologie

5.2 Erfahrungen und Wirkungen auf Gemeinderatsebene

5.3 Erfahrungen und Wirkungen in der Stadt Stuttgart

5.4 Gesellschaftliche Auswirkungen 


\begin{abstract}
Kommunalparlamentarier haben einen großen Bedarf an Unterstützung durch Telekooperation. In dem Pilotprojekt Cuparla wurde von 1996 bis 1998 der Unterstützungsbedarf der Gemeinderäte in Stuttgart erhoben, eine Telekooperationsumgebung für sie entwickelt und in die kommunalpolitische Arbeit eingeführt. Dieser Artikel gibt einen Überblick über das Projekt Cuparla, den Bedarf der Gemeinderäte, über innovative Designideen der Cuparla-Software und über die Erfahrungen bei der Einführung.
\end{abstract}

\title{
1 Einleitung
}

Stadträte sind sehr stark zeitlich belastet. Neben dem Hauptberuf sind sie ehrenamtlich bis über 40 Stunden pro Woche für die Kommune tätig. Da sie in der Verwaltung kein Büro haben, müssen sie sich abends und am Wochenende zu Hause auf Ihre Sitzungen und Entscheidungen vorbereiten. Zu dieser Zeit sind in der kommunalen Verwaltung keine Mitarbeiter. Das Projekt Computerunterstützung der Parlamentsarbeit (Cuparla) versetzt sie in die Lage, mit Hilfe eines Notebooks Gemeinderatsarbeit zu Hause und an jedem anderen Ort durchzuführen und dabei auf Informationsbestände der Verwaltung, der Fraktion und von Kollegen zuzugreifen. Im Rahmen dieses Projektes wurde ein modernes Produktbündel, bestehend aus Telekommunikationsdiensten, Softwareprodukten, Beratungsdienstleistungen und sonstige Dienstleistungsprodukten entwickelt. Cuparla wurde in einem Pilotprojekt mit dem Gemeinderat Stuttgart von Anfang 1996 bis März 1998 erprobt. Es wurden 56 von 57 aktiven Stadträten ausgestattet ${ }^{1}$. In einem zweiten Feld in Kornwestheim wurden 20 von 26 Stadträten im Herbst 1997 ausgestattet, um den Nutzen auch für kleinere Gemeinden zu evaluieren.

Im Folgenden werden zuerst die Ziele des Projekts Cuparla vorgestellt. Danach wird der Bedarf für eine Unterstützung aus der zeitlichen Belastung der Stadträte, ihrer Mobilität und der Informations- und Kooperationsintensität ihrer Arbeit abgeleitet. Sodann wird die Cuparla-Software als ein besonders innovatives Produkt vorgestellt, welches im Rahmen des Projekts entwickelt wurde. Zum Abschluß wird auf die Erfahrungen und Wirkungen eingegangen. Dieser Artikel kann nur einen Überblick geben. Das Pilotprojekt Cuparla wird ausführlich von Schwabe (1998) vorgestellt.

1 Insgesamt hat der Stuttgarter Gemeinderat 60 Mitglieder. Zwei Stadträte waren durch langdauernde Erkrankungen verhindert; ein Stadtrat hatte sich beruflich so weit reorientiert, daß er nicht mehr aktiv an der Fraktions- und Ausschußarbeit teilnahm. Diese drei nichtaktiven Stadträte wurden nicht ausgestattet. 


\section{Ziele des Projekts CUPARLA}

Im August 1995 gab die Deutsche Telekom Berkom GmbH einem Konsortium, bestehend aus der Universität Hohenheim (Projektkoordinator), der Datenzentrale Baden-Württemberg und ITM GmbH im Rahmen des Telearbeitsprogramms der Deutschen Telekom Berkom $\mathrm{GmbH}^{2}$ und der Deutschen Telekom den Auftrag, Telearbeit durch die Computerunterstützung der Gemeinderatsarbeit in einem Pilotprojekt bereitzustellen, zu verwenden und zu evaluieren. Als Anwender wurden die Stadt Stuttgart und die Gemeinde Kornwestheim ausgewählt.

Die Zusammenarbeit der Stadträte soll untereinander und mit der Verwaltung in drei Telearbeitsszenarien unterstützt werden:

- Computerunterstützte Sitzungen zur gleichen Zeit am gleichen Ort: Innerhalb des üblichen Plenarsaals werden beispielsweise Werkzeuge zum Abstimmen und zum gemeinsamen Bearbeiten von Dokumenten, zusätzliche Kommunikationskanäle, Unterstützung für parlamentarische Prozeduren, Zugang zu externen Informationen und ein automatisches Protokollieren von Sitzungsergebnissen in einem computergestützten Parlamentsarchiv zur Verfügung gestellt.

- Telekooperation zur gleichen Zeit an verschiedenen Orten: Den Parlamentsmitgliedern werden Werkzeuge zur Verfügung gestellt, mit denen sie über Kommunikationsverbindungen und spezielle Werkzeuge zusammenarbeiten können.

- Telekooperation zu verschiedenen Zeiten an verschiedenen Orten: Ausschüsse, Fraktionen, die Verwaltung und interessierte Externe arbeiten zusammen, indem sie untereinander Nachrichten austauschen und gemeinsam Dokumente bearbeiten. Alte Dokumente, Sitzungsprotokolle und aufgezeichnete wichtige Sitzungsepisoden werden in einem Sitzungsarchiv abgelegt.

Die Unterstützung umfaßt

- Sitzungsarbeit (Sitzungsvorbereitung, -durchführung und -nachbereitung),

- Ad-hoc-Zusammenarbeit und -Abstimmung zwischen den Sitzungen,

- Teleheimarbeit auf einem gemeinsamen Dokumentenbestand.

Aus diesen Einsatzszenarien läßt sich folgende Ausstattung schließen: Jeder Stadtrat wird mit einem Notebook ausgestattet. Dieses Notebook unterstützt seine Arbeit über verschiedene Kommunikationskanäle.

2 Die Deutsche Telekom Berkom GmbH ist eine 100\% Tochter der Deutschen Telekom. 


\begin{tabular}{|l|l|l|l|}
\cline { 2 - 4 } \multicolumn{1}{c|}{} & \multicolumn{1}{c|}{ Arbeitsplatz } & \multicolumn{1}{c|}{ Mobil/Lokal } & \multicolumn{1}{c|}{ Mobil } \\
\hline Wo & $\begin{array}{l}\text { zu Hause, } \\
\text { in der Verwaltung }\end{array}$ & im Sitzungssaal & überall \\
\hline Wie & ISDN & mobiles LAN & $\begin{array}{l}\text { Mobiltelefonnetz/ } \\
\text {-datennetz }\end{array}$ \\
\hline Was & $\begin{array}{l}\text { Notes, Mail, Sprache, } \\
\text { Video, CATeam, Fax, } \\
\text { Datenbanken, Office }\end{array}$ & $\begin{array}{l}\text { CATeam }{ }^{3}, \text { Notes, } \\
\text { Datenbanken, } \\
\text { Office }\end{array}$ & $\begin{array}{l}\text { Mail, Sprache, Notes, } \\
\text { Office }\end{array}$ \\
\hline
\end{tabular}

\section{Abbildung 1: Telekooperationsunterstuitzung}

Dadurch soll für den Anwender

- die Gemeinderatsarbeit effizienter und flexibler gestaltet,

- der Informationszugang der Stadträte verbessert und

- Kommunikations- und Kooperationsbarrieren innerhalb des Parlaments und zwischen Parlament und Verwaltung abgebaut werden.

Das Pilotprojekt wurde im März 1998 abgeschlossen und in Stuttgart und Kornwestheim in den Regelbetrieb überführt. Dieser Bericht faßt die Projektergebnisse bis einschließlich März 1998 zusammen.

\section{Gemeinderatsarbeit}

Die Parlamentsarbeit in Stuttgart umfaßt wie in anderen Stadtkreisen dieser Größe verschiedenste Gruppen und Akteure, die zum Zwecke der politischen Arbeit miteinander kooperieren. An oberster Stelle steht die Vollversammlung des Stadtrates, die nur zu den wichtigsten Themen einberufen wird sowie eine große Anzahl von Fachausschüssen, deren wichtigster der Verwaltungsausschuß darstellt. Die Informationen, die man benötigt, um in solchen Ausschüssen als Stadtrat kompetent mitentscheiden zu können, werden dabei aus den verschiedensten Quellen bezogen. Eine wesentliche Keimzelle der politischen Arbeit des Stadtrates ist dabei seine eigene Fraktion, wo er sich mit Kollegen der gleichen Partei zu bestimmten Gelegenheiten (Fraktionssitzungen, Arbeitskreissitzungen) trifft, um den politischen Kurs abzugleichen. Basis dieser Arbeit bildet dabei das Geschäftszimmer der Fraktion, welches ihm die zur politischen Debatte notwendigen Informationen recherchiert, sammelt, aufbereitet und zukommen läßt. Um also einen umfassenden Überblick über die Koopera-

3 CATeam unterstützt Sitzungen mit Computern nach dem Vorbild der Metaplantechnik. Zu CATeam vergleiche (Lewe 1995) und (Schwabe 1995). 
tionsprozesse der Stadträte zu erhalten und damit zu erfahren, wer mit wem auf welche Weise und zu welchem Zweck kooperiert, und wie diese Arbeitsweise sich auf den politischen Entscheidungsprozeß auswirkt, war es für uns erforderlich, alle bereits aufgezählten Akteure, Veranstaltungen und Gruppen in ihrer Arbeitsweise und -funktion im Gesamtsystem zu erforschen.

\subsection{Dauer der Gemeinderatsarbeit}

Stadträte üben ihr Amt nebenberuflich aus. Die folgende Abbildung zeigt, daß der Stadtrat im Durchschnitt deutlich mehr Zeit für sein Ehrenamt verwendet als für seinen Hauptberuf.

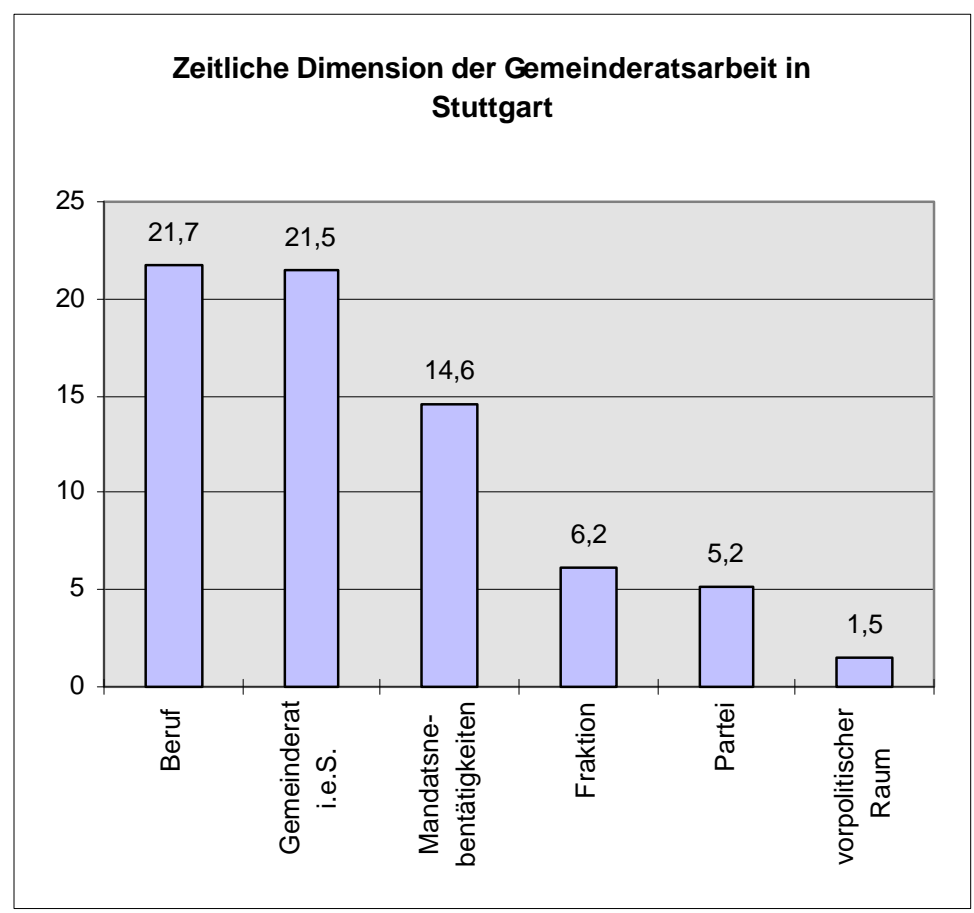

Abbildung 2: Arbeitszeit eines Stadtrats pro Woche

Allein schon mit der Gemeinderatsarbeit im engeren Sinne, also insbesondere mit den Sitzungen, ist er mit ca. 20 Stunden pro Woche ungefähr genauso lang beschäftigt, wie mit seinem Hauptberuf. Ein weiterer großer Block sind Mandatsnebentätigkeiten wie Bürgerkontakt, auswärtige Repräsentation oder Informationssammlung. Zusammen mit der Fraktionsarbeit, der Parteiarbeit und der Arbeit im vorpolitischen Raum (z.B. Vereine) ergibt das eine durchschnittliche zeitliche Belastung von 42,3 Stunden pro Woche. Ein bedeutender Teil dieser Arbeit wird in den Abendstunden und an den Wochenenden geleistet. Diese 
Daten wurden durch einwöchige Zeitanalysen im Frühjahr 1996 erhoben: 16 Stadträte protokollierten ihren Zeitaufwand in Halbstundenblocks, 29 rekonstruierten eine Woche anhand ihres Terminkalenders (für Details der Erhebung vgl. Schwabe 1998 und Vöhringer 1996).

\subsection{Kooperationspartner der Gemeinderatsarbeit}

Der einzelne Stadtrat ist für seine Gemeinderatsarbeit in ein umfassendes Netzwerk von Kooperationsbeziehungen eingebettet. Die Kooperationsbeziehungen lassen sich den drei Bereichen "Gemeinderat", "Verwaltung" und "Umfeld" zuordnen (vgl. Abbildung 3).

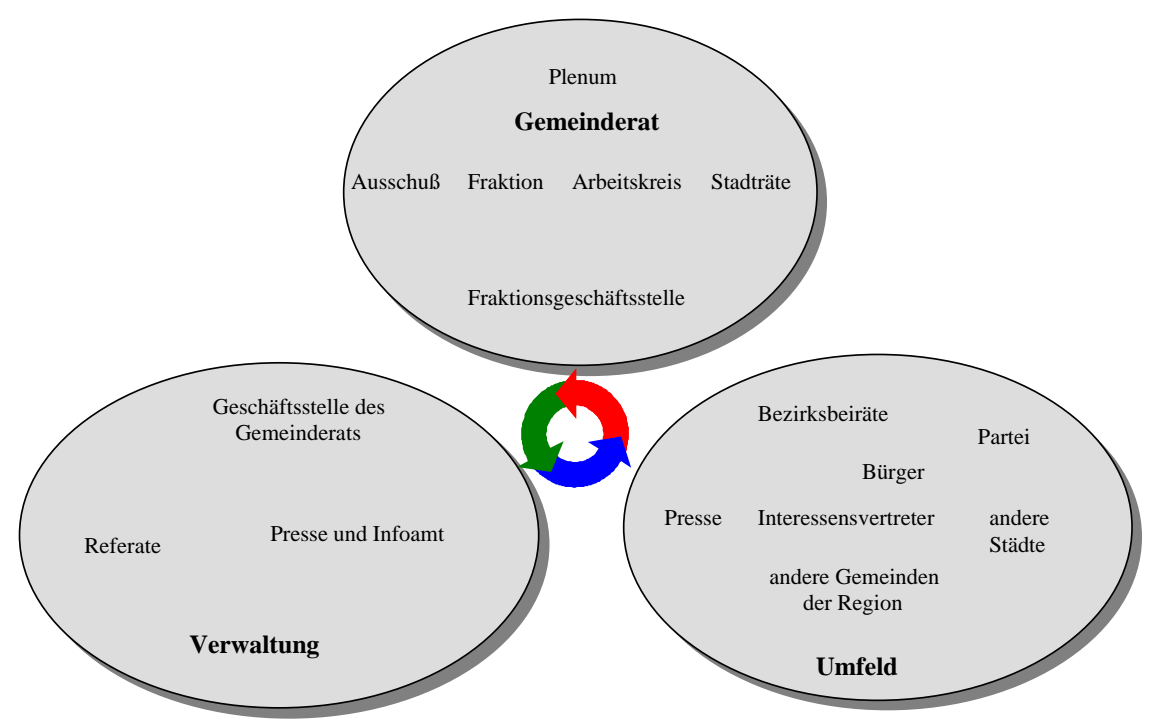

Abbildung 3: Kooperationspartner der Gemeinderatsarbeit

Innerhalb des Gemeinderats arbeitet der einzelne Stadtrat im Plenum, in den einzelnen Ausschüssen, in Arbeitskreisen und mit einzelnen anderen Stadträten zusammen. Die Fraktionsgeschäftstelle ist eine Schnittstelle sowohl zur Verwaltung als auch zum Umfeld.

Der Stadtrat steht in Kooperationsbeziehungen zu seinem politischen Umfeld. Der direkte Kontakt zum Bürger ist dem Stadtrat wichtig, weil er sich auch als „Vertreter des kleinen Mannes" gegenüber der Verwaltung versteht. Die Bezirksräte dienen als weiteres Ohr an der Basis. Mit seiner Partei steht er insbesondere zu Wahlkampfzeiten im engen Kontakt. Interessenvertreter wie Bürgerinitiativen, Gewerkschaften oder Wirtschaft treten an ihn heran, um ihren Anliegen Gehör zu verschaffen. Mit anderen Gemeinden der Region versucht er Koalitionen zu bilden, um gemeinsam Anliegen voranzubringen und von anderen vergleich- 
baren Großstädten versucht er zu lernen. Die Presse gibt immer wieder Anstöße für Initiativen der Stadträte.

In der Verwaltung versorgt die Geschäftsstelle des Gemeinderats die Stadträte mit Informationen und Beschlußvorlagen und nimmt die Anfragen und Anträge der Stadträte entgegen. Die Referate mit den Bürgermeistern als Referatsleitern sind die politischen Ansprechpartner in der Verwaltung. Das Presse- und Informationsamt verbreitet die Beschlüsse in der Öffentlichkeit.

Zwei Projekte sind für die Unterstützung des Gemeinderats von unmittelbarem Belang (vgl. Abbildung 4): Das Projekt "Kommunaler Sitzungsdienst" unterstützt die Vorlagenerstellung, Antragsbearbeitung und Informationsbereitstellung in der Verwaltung. Das Projekt CUPARLA unterstützt die Zusammenarbeit und Informationsversorgung im Gemeinderat. Beide Projekte kümmern sich gemeinsam um die Schnittstellen zwischen Verwaltung und Gemeinderat.

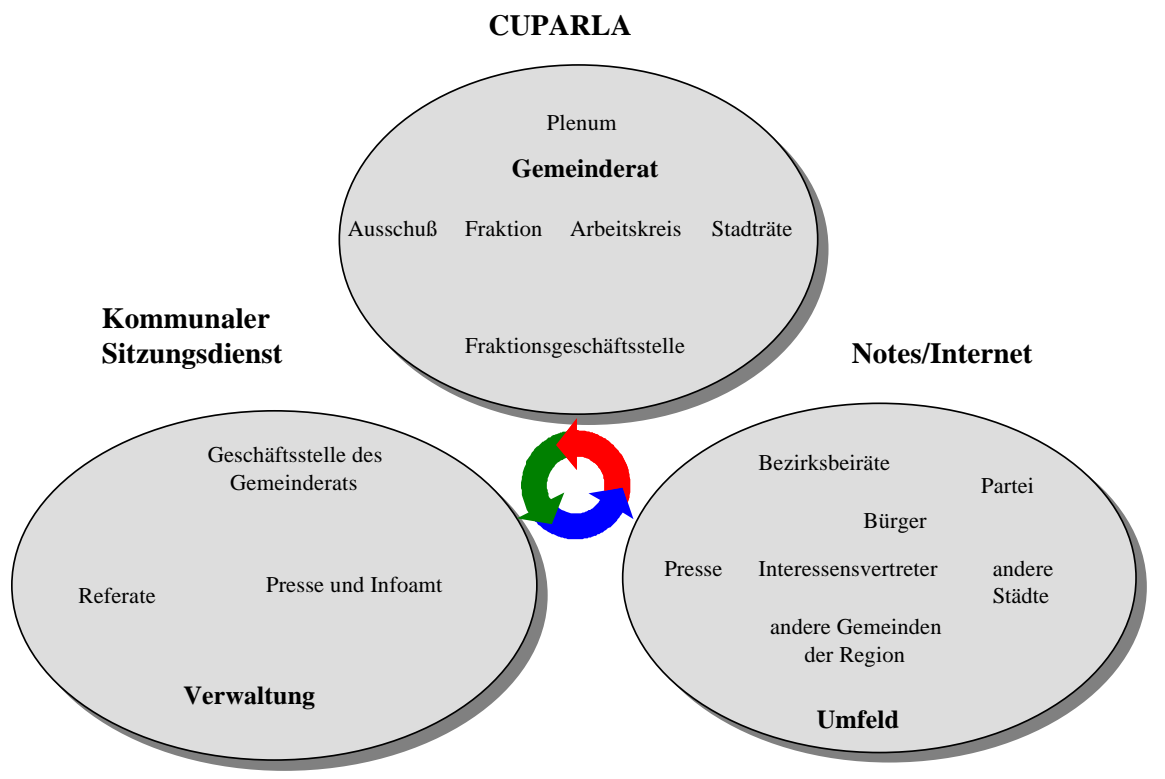

Abbildung 4: Projekte und Projektansätze

Für die Schnittstelle zum Umfeld des Gemeinderats (und der Verwaltung) sind in Stuttgart Projekte mit Notes/Internet-Kopplungen in Arbeit.

\subsection{Orte der Gemeinderatsarbeit}

Ein Stadtrat hat keinen festen Arbeitsplatz, sondern ist an mehreren Orten tätig. Diese Orte lassen sich in die Bereiche Verwaltung, entfernter fester Arbeitsplatz und mobiler Arbeitsplatz einteilen (vgl. Abbildung 5). 


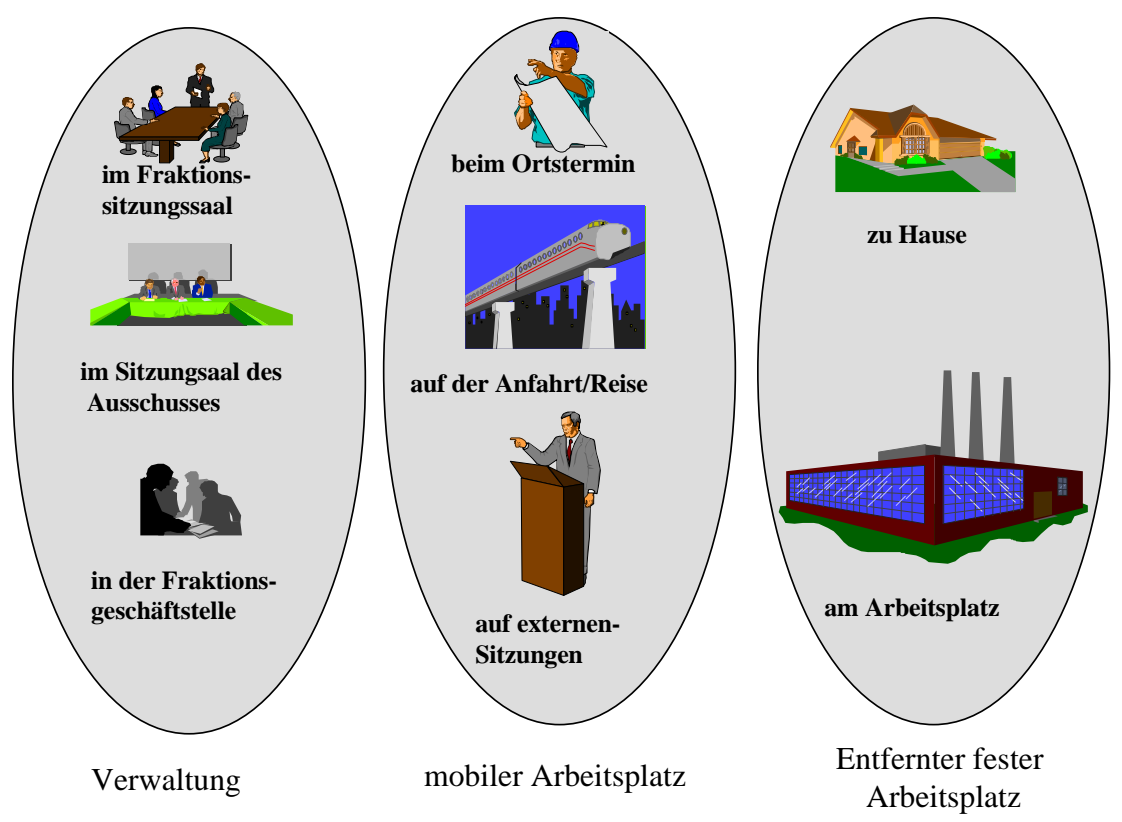

Abbildung 5: Orte der Gemeinderatsarbeit

In der Verwaltung bespricht er sich mit seinen Fraktionskollegen im Fraktionssitzungssaal um die Fraktionspolitik festzulegen. In den Ausschüssen faßt er mit Vertretern (oder gegen Vertreter) anderer Parteien die meisten Beschlüsse zur politischen Sacharbeit. Die Fraktionsgeschäftstelle koordiniert die Arbeit der Stadträte, versorgt sie mit Informationen und übernimmt Sekretariatsarbeiten. Die überwiegende Mehrheit der Stadträte hat aber keinen eigenen Arbeitsplatz in der Verwaltung.

Für die eigene Sacharbeit (Lesen von Vorlagen, Verfassen von Anträgen etc.) ist der einzelne Stadtrat auf sein Büro zu Hause angewiesen. Dies ist auch deshalb kaum zu ändern, weil die Sacharbeit häufig an Wochenenden sowie zu den Abendstunden durchgeführt wird. Zu Hause befindet sich auch das persönliche Archiv jedes Stadtrats. Wenn der Stadtrat freiberuflich tätig ist (und das sind vergleichsweise viele), dann führt er einen Teil seiner Arbeit an seinem beruflichen Arbeitsplatz durch. Der Stadtrat ist auch immer wieder unterwegs tätig. Er nimmt Ortstermine war, um sich vor Ort zu informieren (z.B. bei Bauvorhaben) oder um die eigene Fraktion politisch zu repräsentieren. Für jeden Stadtbezirk stellen die großen Fraktionen eigene Bezirksbetreuer ab, die als Schnittstelle zu den lokalen Parteivertretern, Bürgern und Bezirksbeiräten dienen. 


\section{Die Cuparla-Software}

In der traditionellen Entwicklung von Groupwareanwendungen werden zwei verschiedene Ansätze verfolgt: Die Orientierung an dem Arbeitsfluß wird im folgenden der "prozeßorientierte Ansatz" genannt, die Orientierung an den Arbeitsdokumenten "materialorientierter Ansatz". In den folgenden Absätzen zeigen wir zuerst auf, warum beide für die Unterstützung der Gemeinderatsarbeit nicht gut geeignet sind; dann zeigen wir, wie mit einem "kontextorientierten" Ansatz eine geeignete Unterstützung geliefert werden kann.

\subsection{Prozeßorientierter Ansatz}

Der prozeßorientierte Ansatz nimmt den Fluß der Dokumente durch eine Organisation zum Ansatzpunkt für eine Unterstützung. Unter dem Stichwort "Workflowsysteme" werden Programmpakete angeboten, die es einzelnen Beteiligten erlauben, den Fluß von Dokumenten zu steuern. Der Prototyp eines Workflows ist eine Schadensfallbearbeitung in einer Versicherung, die immer nach dem gleichen Muster abgewickelt wird. Ziel dieser Systeme ist es, die Liegezeiten zu reduzieren, die Koordination der Arbeitsschritte durch Automatisierung zu erleichtern und den Arbeitsfortschritt für die Vorgesetzten einfacher kontrollierbar $\mathrm{zu}$ machen. Im folgenden wird mit einem "starren Workflow" argumentiert. In der Literatur werden auch "flexible Workflowsysteme" diskutiert (vgl. Weiss \& Krcmar 1996), bei denen aber in abgeschwächtem Maße die gleichen Probleme auftreten.

Kernvoraussetzung für eine Unterstützung ist die Gleichartigkeit, Wiederholung und Routinisierbarkeit der Aufgabe. Dies ist bei der Gemeinderatsarbeit aber nur vordergründig der Fall: Zwar lassen sich einzelne Geschäftsprozesse identifizieren, z.B. "Antrag stellen" oder "Haushalt beraten"; aber im Detail wird jeder Antrag anders gestellt, verläuft jede Haushaltsberatung anders. Es ist in der Praxis noch nicht einmal festgelegt, welche Personen an welchen Geschäftsprozessen mitwirken, z.B. weil jeder Stadtrat von sich aus mit einem Antrag initiativ werden kann und mit jedem anderen Stadtrat eine Koalition eingehen kann. Ein konkretes Beispiel: In einer Fraktion des Stuttgarter Gemeinderats besteht der Fraktionsvorsitzende darauf, daß alle Anträge aus seiner Fraktion vorher von ihm abgesegnet werden müssen. Einzelne Stadträte bestehen wiederum darauf, daß sie auch ohne Zustimmung ihres Vorsitzenden Anträge stellen dürfen und tun dies. Sobald ein Workflowsystem eine "richtige" Vorgehensweise vorschreibt, werden die Benachteiligten versuchen, das Workflowsystem zu umgehen und die Nutzung zum Erliegen bringen. Da der Stadtrat aus der Eigenverantwortlichkeit und aus dem Initiativrecht seine wesentliche Motivation und Macht zieht, steht er allen ihn beschränkenden Regeln der Zusammenarbeit sehr skeptisch gegenüber. Auch von der Sache her sind die Entscheidung über die Belange einer Stadt zu anspruchsvoll, zu komplex und zu vielfältig, als daß sie in einem routinisierten Arbeitsprozeß bearbeitet werden können. 


\subsection{Materialorientierter Ansatz}

Gryczan und Züllighoven (1992) schlagen vor, qualifizierte Arbeit nicht durch Vorstrukturierung, sondern durch die Bereitstellung von geeigneten Werkzeugen und Materialien zu unterstützen. Materialien sind in einer ersten Annäherung Dokumente; Werkzeuge sind Editoren, mit denen die Dokumente bearbeitet werden können. Dies kommt dem Gedanken einer freien Arbeitsumgebung in erster Annäherung auch entgegen. In einem ersten Entwurf der Cuparla-Software wurde deshalb dieser Weg verfolgt. Für die Stadträte hieß das, daß ihnen Anträge, Vorlagen, Presseerklärungen, Freitextdokumente etc. in einer Dokumentendatenbank zur Verfügung standen. Der Vorteil dieser Vorgehensweise war es, daß Informationen leicht automatisch gesucht werden konnten, weil sie sich nur in einer Datenbank befinden konnten.

Der Verzicht auf jede Vorgabe eines Arbeitsprozesses kam den Stadträten auch entgegen. Da aber überhaupt keine Struktur vorgegeben wurde, wurde die Koordination der Zusammenarbeit sehr schwierig. Insbesondere die Vergabe von Berechtigungen zum Zugriff auf Dokumente wurde kompliziert. Die typische Entstehung eines Antrags soll dies verdeutlichen:

Der Anstoß zu einem Antrag wird häufig von einem Bürger an einen einzelnen Stadtrat herangetragen. Dieser entwirft dann für sich eine Skizze. Dann spricht er diese Skizze mit befreundeten Kollegen ab und bringt sie in die Fraktion ein. Dort wird sie durchgesprochen, möglicherweise überarbeitet und als Antrag an die Verwaltung weitergegeben. In der Verwaltung wird eine Stellungnahme der Verwaltung erstellt und in einer Ausschußsitzung den Stadträten mitgeteilt. Sodann wird in dem Ausschuß über den Antrag abgestimmt. Der Antrag durchläuft also verschiedene Stadien der Vertraulichkeit. Um diese Vertraulichkeit sicherzustellen, müßte der Stadtrat bei Verwendung nur einer Datenbank für jedes Dokument einzeln Zugriffsrechte vergeben und im Laufe der Beratungen verwalten. Dies ist jedoch bei der Menge der verwalteten Dokumente und der Komplexität der Abläufe eine unrealistische Erwartung.

Der materialorientierte Ansatz hat sich zu weit von der Gemeinderatsarbeit entfernt; Gemeinderatsarbeit wird nicht im wesentlichen durch die verwendeten Materialien geprägt, sondern dadurch, wer sich mit wem verbündet, um seine Ziele zu erreichen. Für diese Bündnisse gibt es mit den Fraktionen, Arbeitsgruppen und (in Grenzen) Ausschüssen tradierte Standardvorgaben. Sie sind in dem zuerst verfolgten materialorientierten Ansatz zu sehr in den Hintergrund gerückt.

\subsection{Kontextorientierter Ansatz}

Die Analyse hatte gezeigt, daß sich Stadträte in verschiedenen Arbeitskontexten verschieden verhalten: Während sie mit Kollegen oder in der eigenen Fraktion noch relativ offen und gelöst sind, dominiert in den Ausschüssen und im Gesamtgemeinderat die Auseinandersetzung mit dem politischen Gegner. Diese verschiedenen Arbeitskontexte bestimmen auch das Kooperationsverhalten und den 
Informationsaustausch zwischen den Stadträten. Deshalb wurde beschlossen, die Arbeitskontexte zum Kern des Softwareentwurfs zu machen. Erleichternd kam hinzu, daß sich jeder Arbeitskontext eines Stadtrats leicht durch einen Raum symbolisieren läßt: Das Fraktionszimmer steht für den Arbeitskontext "Fraktion", das Arbeitszimmer für den Arbeitskontext "zu Hause", der Ausschußsitzungssaal für den Arbeitskontext "Ausschuß" etc. Durch die Verwendung einer Raummetapher lassen sich bisher schon praktizierte Verhaltensweisen auf den Umgang mit der Software übertragen. Es ist einem Stadtrat intuitiv einsichtig, daß ein Dokument allen Fraktionskollegen zugänglich ist, sobald er es in das Fraktionszimmer verschiebt.

In den verschieden Räumen befinden sich schon heute Hilfsmittel, die die Zusammenarbeit strukturieren. Wir haben diese Hilfsmittel "Dokumentenaufbewahrungsorte" genannt. Befindet sich ein Dokument (oder ein ganzer Vorgang) auf dem Dokumentenaufbewahrungsort "Schreibtisch", dann bedeutet dies, daß der Schreibtischbesitzer dieses Dokument von sich aus bearbeitet, ohne daß es eines weiteren Anstoßes von außen bedarf. Befindet sich das Dokument in der "Ablage", dann ist das behandelte Thema zwar noch aktuell, es wird aber erst herangezogen, wenn von außen hierzu ein Anstoß kommt. Im "Archiv" befinden sich die erledigten Vorgänge, auf dem "Sitzungstisch" die Unterlagen für die nächste Sitzung und am schwarzen Brett "hängen" Bekanntmachungen. Für einzelne Personen mögen einheitliche Dokumentenaufbewahrungsorte überflüssiger Ballast sein; für die Koordinierung der Zusammenarbeit sind sie aber wichtig, da sie zu einheitlichen Erwartungen und abgestimmten Verhaltensweisen führen. Deshalb haben wir diese (und andere) Dokumentenaufbewahrungsorte direkt in das Softwaredesign übernommen.

Am Beispiel des Antrags läßt sich ein typischer Arbeitsprozeß in den Räumen erläutern (dies ist kein Workflow, sondern nur eine von vielen plausiblen Möglichkeiten!): Der Stadtrat erstellt in seinem privaten elektronischen Arbeitszimmer eine erste Fassung. Dann nimmt er ihn mit in die Arbeitsgruppe, indem er es in das Arbeitsgruppenzimmer verschiebt. Sobald die Arbeitsgruppe das Dokument gemeinsam fertiggestellt hat, wird es in die Fraktion in das Postfach des Fraktionsvorsitzenden verschoben. Wenn dieser den Antrag abgezeichnet hat, legt ihn er selbst oder der Fraktionsassistent aus dem Postfach auf den Sitzungstisch im Fraktionszimmer. Sobald die Fraktion in der darauffolgenden Fraktionssitzung von dem Antrag Kenntnis genommen hat, wird er in dem Verwaltungsraum abgelegt. Von dort wandert er über die Verwaltung in die Ausschüsse und für Protokoll und Ablage zurück in die Verwaltung. In den Arbeitskontexten werden somit die gemeinsamen und privaten Dokumente adäquat verwaltet. Ferner lassen sich Arbeitsprozesse so durchführen, wie sie heute auch schon durchgeführt werden. 


\subsection{Umsetzung}

Abbildung 6 zeigt die Cuparla-Eingangshalle mit den Räumen, die den einzelnen Stadträten zur Verfügung gestellt werden. Jeder Raum hat seine eigene Zugangsberechtigung. "Betritt" ein Stadtrat das Arbeitszimmer, befindet er sich in seinem individuellen Arbeitskontext, zu dem nur er Zugang hat; der Raum "Fraktion" steht für seine eigene Fraktion; die anderen Fraktionen sind für ihn unsichtbar. $\mathrm{Zu}$ dem "Gemeinderat" haben alle Stadträte Zugang; in der "Verwaltung" und der "Bibliothek" sind alle Dokumente abgelegt, die für Mitarbeiter der Stadt und für die Stadträte offen sind usw.

Die Abbildung 7 zeigt das elektronische "Fraktionszimmer": Postfächer, Ablage, Archiv und Tische sind dem physischen Fraktionszimmer nachgebildet. Links sind die Dokumentenaufbewahrungsorte und rechts die Kategorien von Dokumenten des ausgewählten Ortes zu sehen. Unter jeder Kategorie können dann Formulare verschiedenen Typs (Anträge, ...) sowie Office-Dokumente erzeugt und abgelegt werden. Um die Bedienung so klar wie möglich $\mathrm{zu}$ halten, wurde bewußt auf möglicherweise verwirrende besondere grafische Effekte verzichtet. Anhand der Screenshots wird ersichtlich, wie der Arbeitskontext direkt als Strukturierungs- und Orientierungsinstrument für die Anwendung verwendet werden kann.

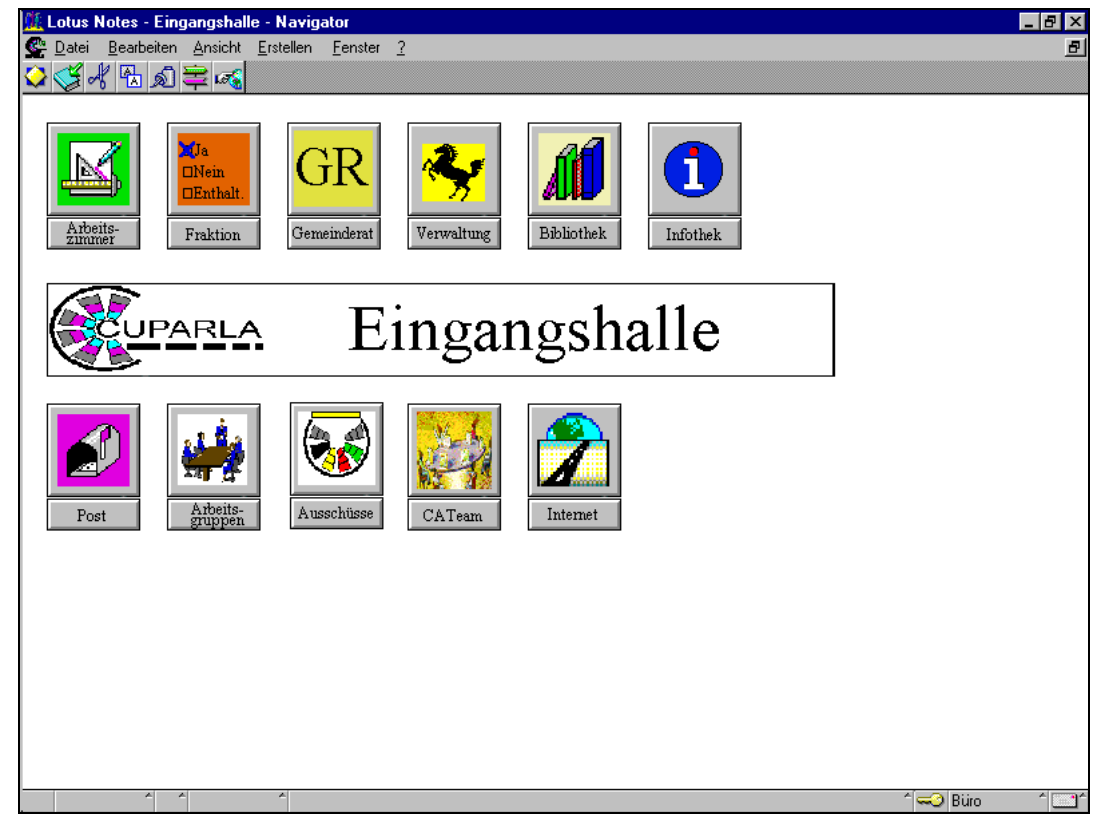

Abbildung 6: Die Cuparla-Eingangshalle 


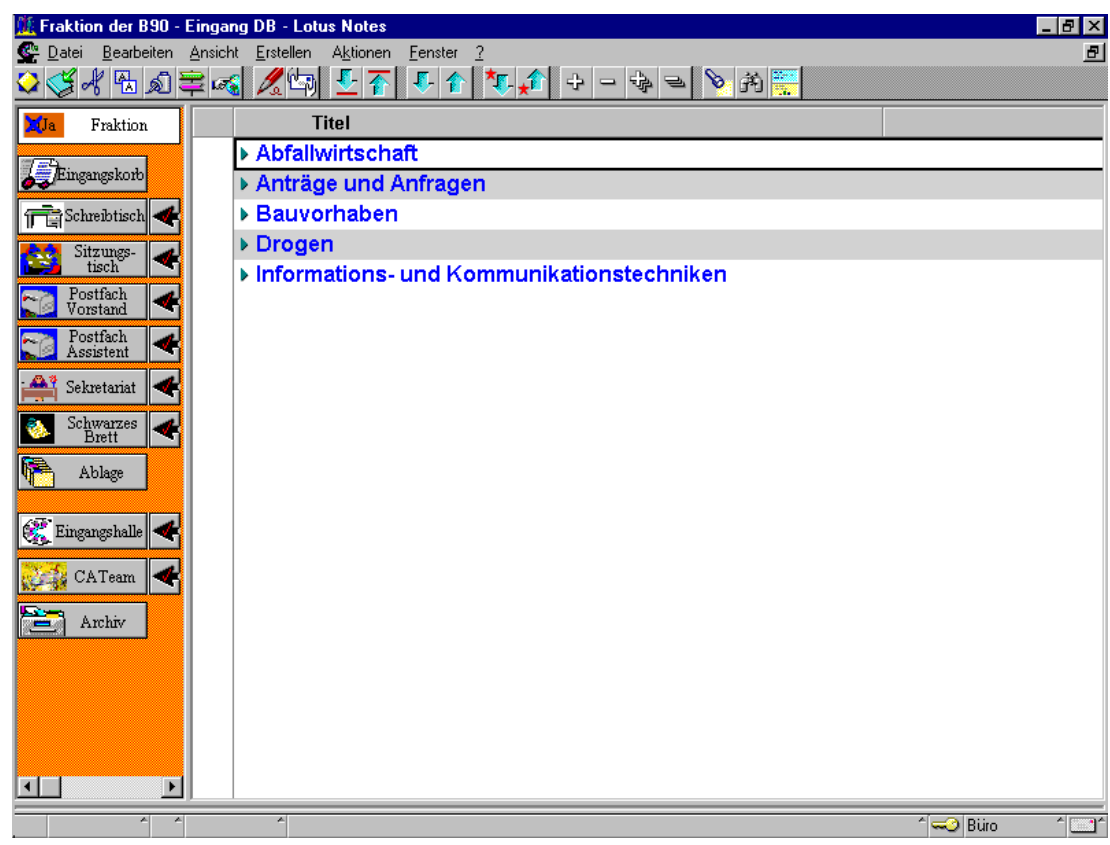

Abbildung 7: Das Cuparla-Fraktionszimmer

Was ist neu an dem Ansatz der Cuparla Software? Die Raummetapher wurde schon von Henderson \& Card (1986) für die Gestaltung von Benutzerschnittstellen eingeführt. Cuparla Räume sind nicht nur eine Interfacemetapher, sondern dienen auch als Dokumentenaufbewahrungsorte und als Kontext mit spezifischen Zugangsprivilegien und -restriktionen, und, als wesentlichster Punkt, bieten sie den verschiedenen politischen Kulturen Raum, sich zu entwickeln.

In Cuparla sind Räume auch nicht nur gemeinsame Arbeitsbereiche, wie sie in Pankoke-Babatz \& Syri (1996) diskutiert werden. Raum ("Space", also hier nicht in der Bedeutung von Zimmer!) ist eine häufig verwendete Metapher für den gemeinsamen Bereich, der Zusammenarbeitenden zur Verfügung gestellt wird. Er wird insbesondere im Zusammenhang mit Arbeit als "Workspace" oder "Media Space" bezeichnet. In einem dreidimensionalen Raum gibt es Nähe und Ferne, vorn und hinten und rechts und links. In einem Media-Space oder Workspace werden diese Eigenschaften von Raum verwendet, um Arbeitsmaterialien und die Zusammenarbeitenden anzuordnen und den Kooperierenden Orientierung zu bieten (Benford et al. 1996).

Cuparla-Räume sind eher "Plätze der Zusammenarbeit". Forscher von Xerox Parc argumentieren, daß nicht "Raum" ("Space") wesentlich für Orientierung von Akteuren ist, sondern "Plätze" (places) (Harrison \& Dourish 1996). Plätze, als Gegenden, Räume und Orte in einem Raum, haben eine bestimmte Bedeutung und mit ihnen werden bestimmte Umgangsformen verbunden. In einer Teeküche 
verhält sich eine Person anders als in ihrem Büro oder im Kaufhaus. Sie betonen deshalb die Notwendigkeit, die physischen Plätze, an denen Telekooperation stattfindet, als Kontext bewußt in die Gestaltung von Telekooperationssystemen mit einzubeziehen.

Ein Cuparla-Raum enthält mehrere gemeinsame Arbeitsbereiche mit spezifischer sozialer Bedeutung. Diese erlauben die Etablierung spezifischer Regeln und Konventionen für alle Arten der Zusammenarbeit innerhalb einer großen Gruppe. Die Räume in Cuparla bieten genug Struktur zur Orientierung und zur Etablierung von Regeln und genug Flexibilität, daß die Stadträte von einem Arbeitskontext zum anderen wechseln können. Bei der Analyse der Nutzung zeigt sich auch, daß diese Flexibilität durch die Stadträte genutzt wird: In Abhängigkeit von den Gepflogenheiten und dem Vertrauen der Fraktionsmitglieder werden die gleichen Räume von den unterschiedlichen Fraktionen verschieden genutzt. Während eine Fraktion ihr Fraktionszimmer zur Koordination von Aktivitäten und zur Verbreitung von Informationen zu abgeschlossenen Vorgängen verwendet, werden in einer anderen Fraktion auch unfertige Angelegenheiten im Fraktionszimmer offen diskutiert.

\subsection{Weitere Produkte}

Spätestens seit der Verbreitung der SAP-Software R3 ist bekannt, daß die Wertschöpfung von Software für Organisationen und Organisationseinheiten in der begleitenden Beratung, Schulung, Konfiguration sowie in speziell dafür erstellten Telekommunikationsdienstleistungen und dem Betrieb eines Dienstes besteht. Deshalb gehen Anbieter dazu über, Dienstleistungen und Dienste als Produkte zu vermarkten und zu Produktbündeln zusammenzufassen. Ein solches Produktbündel wird auch im Cuparla-Projekt und im Anschluß an das Cuparla-Projekt entwickelt. Im Kern des Produktbündels steht die Cuparla-Software mit ihren Komponenten für den Anwender und für den Server sowie deren Bereitstellung als Dienst auf "Cuparla-Serverfarmen". Die Cuparla-Software stellt mit den "Räumen" eigene Groupware-Funktionalitäten für die Dokumentenverwaltung, die Kommunikation und die Kooperation zur Verfügung. Sie ist gleichzeitig Ankerpunkt für die Integration weiterer Dienste und Produkte wie das Internet, GroupSystems für die Sitzungsunterstützung, Videoconferencing und eine FaxAnbindung.

Diese Software wird den Stadträten auf einem speziell konfigurierten "Telekooperationsnotebook" ausgeliefert. Die Applikation soll in naher Zukunft über "Notesserverfarmen" der Deutschen Telekom bundesweit zur Verfügung gestellt werden. Diese Serverfarmen kommunizieren mit lokalen Servern in den Kommunen. Für die bedarfsgerechte Gestaltung entwickelte die Universität Hohenheim mit dem "Needs Driven Approach" eine eigene Analyse und Designmethode (Schwabe \& Krcmar 1996) sowie eine Methode zur Einführung und Evaluation von Telekooperation (Schwabe 1998). 


\section{Erfahrungen und Wirkungen}

\subsection{Akzeptanz der Technologie}

Seit der Sommerpause 1997 sind praktisch alle Stuttgarter Stadträte mit Notebooks ausgestattet, greifen auf die zentral vorgehaltenen Informationsbestände zu, kommunizieren über Email und kooperieren über gemeinsame "Räume". Die Akzeptanz der Technologie hängt von einer geeigneten Kombination von Technik und Einführungsmaßnahmen ab.

Die Akzeptanz steigt in dem Maße, in dem die Verwaltung für die Stadträte relevante Informationen bereitstellt und mit der Anzahl der ausgestatteten Stadträte. Bis März 1998 wurden über 8500 Anträge, Vorlagen und Protokolle sowie das gesamte Stadtrecht, der Haushalt und das Telefonbuch der Stadtverwaltung Stuttgart erfaßt.

Die Akzeptanz steigt weiterhin in dem Maße, in dem die Arbeit der Stadträte umfassend abgedeckt wird. Hierzu gehören beispielsweise die Integration von Fax, Internet und PC-Office-Umgebung. Sie ist wichtiger als eine besonders ausgefeilte Bereitstellung von Einzelfunktionalitäten.

Zum dritten steigt die Akzeptanz mit den Kenntnissen der Stadträte im Umgang mit Notebook und Software. Je mehr Stadträte ausreichend Kenntnisse haben und Cuparla regelmäßig nutzen, desto mehr lohnt sich die Nutzung für jeden.

Die Bereitstellung einer bedarfsgerechten Technologie stellt nur den kleineren Teil des Aufwandes für eine erfolgreiche Pilotierung von Telekooperation dar. Der Hauptaufwand liegt in der geschickten Einführung. In der Endbefragung sahen die Stadträte in der proaktive Unterstützung durch die Implementierer einen Hauptgrund für die Akzeptanz von Cuparla. Von besonderer Bedeutung sind Schulungen, die sich an der Arbeit der Gemeinderäte orientieren und nicht an den Funktionalitäten der Software (z.B. "Wie schreibe ich mit meinen Kollegen einen Antrag?" und nicht "Wie funktioniert der Cuparla-Editor?"). Die Stadträte führen aus Zeitgründen die Schulungen ganztags am Wochenende durch.

$\mathrm{Zu}$ der Implementierung gehört auch die Anpassung der Arbeitsprozesse in den Fraktionen, im Gemeinderat und in der Verwaltung. Allein schon die digitale Bereitstellung der Unterlagen bedeutet für die Stuttgarter Verwaltung einen großen Fortschritt. Hierzu sind Implementierer gefordert, die die individuelle und organisatorische Aneignung der Technologie voranbringen. Durch eine Gestaltung der individuellen Aneignung wird ungeeigneten individuellen Nutzungsformen (z.B. Verwendung einer Textverarbeitung zur Tabellenkalkulation oder einer Tabellenkalkulation zur Textverarbeitung) entgegengewirkt. Eine besondere Herausforderung ist die Gestaltung der Aneignung in der Zusammenarbeit. Wann sollte Email verwendet werden und wann gemeinsame digitale Arbeitsbereiche? Wie kann sichergestellt werden, daß jeder Stadtrat regelmäßig seine 
elektronische Post liest, wenn es sich für jeden einzelnen lohnt, zu warten, bis die anderen es tun.

Die Implementierer müssen in Zusammenarbeit mit den Anwendern, der Verwaltungsspitze und Verwaltungsmitarbeitern geplante Veränderungen voranbringen, aber auch sich überraschend ergebende Gelegenheiten wahrnehmen und auf unvorhergesehene Ereignisse reagieren. In Cuparla konnte der Wettbewerb unter den Stadträten und Fraktionen sowie im Verhältnis zur Verwaltung dazu genutzt werden, die Einführung voranzubringen (vgl. Schwabe \& Krcmar 1998).

In Cuparla nahmen die Implementierer weitgehend die Rolle von Moderatoren ein. In einem politisierten Umfeld wie dem Gemeinderat hat nur ein externer Moderator ausreichend Glaubwürdigkeit. Hierbei erwies sich die Universität Hohenheim als Institution ohne eigene politische und ökonomische Interessen als gut geeignet.

In einem Pilotprojekt steht die Einführung in direkter Wechselwirkung mit der Gestaltung der Technologie. Im Kontakt mit den Anwendern ergeben sich Anforderungen, die in die Technologie einfließen und dann wiederum im Feld eingeführt werden müssen.

\subsection{Erfahrungen und Wirkungen auf Gemeinderatsebene}

Kosten und Nutzen wurden im März 1998 aufbauend auf einem Schema von Reichwald et al. (1998) erhoben. Daten wurden von allen 57 aktiven Stadträten (d.h. sowohl Cuparla-Nutzer als auch Nichtnutzer) über einen Fragebogen und offene elektronische Diskussionen zu qualitativen Auswirkungen erhoben: Nach Auskunft von Anwendern führen die zur Verfügung gestellten Informationen jetzt schon zu informierteren Beschlüssen und zu mehr Transparenz der Verwaltungsarbeit. In der Endbefragung gaben die Stadträte als wesentlichen Nutzen die verbesserte örtliche und zeitliche Flexibilität und die durch die verbesserte Informationsbereitstellung verbesserte Qualität der Gemeinderatsarbeit an. Hauptberuf, Mandat und Privatleben sind durch die erhöhte zeitliche und örtliche Flexibilität besser vereinbar geworden. Eine detaillierte Analyse der Auswirkungen ist bei (Schwabe 1998) zu finden.

Ein überraschender Nebeneffekt besteht darin, daß die Stadträte jetzt progressivere Beschlüsse zum Einsatz von Informations- und Kommunikationstechnologie fällen, weil sie die Potentiale der Technologie selbst erleben. Die zentrale Ablage in der Verwaltung und in den Fraktionen führt zu einer deutlichen Arbeitsentlastung der Stadträte. Durch Cuparla werden Mandat und Privatbereich besser miteinander vereinbar und das Gemeinderatsmandat attraktiver. Dies gilt insbesondere für Berufsgruppen, die nicht über die Zeitflexibilität freier Berufe verfügen. 


\subsection{Erfahrungen und Wirkungen in der Stadt Stuttgart}

Das Projekt Cuparla hat zu Innovationen in der Stadtverwaltung Stuttgart geführt. Diese Innovationen haben zum Teil eine Rationalisierungswirkung, zum Teil führen sie zum Aufbau neuer Dienstleistungen.

Die meisten Informationen, die der Gemeinderat für seine Arbeit benötigt, liegen in der Stadt Stuttgart in digitaler Form vor. Allerdings gibt es keinen Standard für die verwendeten EDV-Systeme; vielmehr werden die Daten sowohl auf Großrechnern, als auch auf Workstations und PCs verarbeitet. Es ist durchaus üblich, daß das gleiche Dokument im Laufe seines Wegs durch die Verwaltung mehrmals abgetippt wird. Die Stadt Stuttgart hat sich deshalb dazu entschlossen, das gesamte Vorlagenwesen auf ein einheitliches PC-gestütztes Verfahren umzustellen. Diese Vorlagen und weitere Informationen werden dann allen Ämtern einschließlich der Bezirksämter zur Verfügung gestellt und verringert dort den Such- und Aktualisierungsaufwand für Informationen beträchtlich.

Es ist zu erwarten, daß mit der zunehmenden Einführung der Technologie sich die Abläufe in der Verwaltung verändern und neue Berufsbilder entstehen. Benötigt werden z.B. Sitzungschauffeure, die Technologie in den Gremiensitzungen bereitstellen und Informationsbroker, die die Datenbanken der Verwaltung gezielt für den Gemeinderat durchsuchen und ihm elektronisch aufbereitet zur Verfügung stellen.

Schon heute zeichnet sich ab, daß sich die Unterstützung des Gemeinderats nicht von der Unterstützung der Bürgermeister trennen läßt. Die Bürgermeister haben ein Interesse daran, daß der Gemeinderat keinen Informationsvorsprung hat und drängen darauf, daß sie etwa gleichzeitig wie die Stadträte ebenfalls mit Technologie ausgestattet werden. Da damit von den Sachbearbeitern über die politische Spitze bis zum Gemeinderat alle Beteiligten mit der gleichen technologischen Plattform ausgestattet sind, besteht die Gelegenheit, daß sich die Zusammenarbeit zwischen Politik und Verwaltung grundlegend verbessert. Dies ist das ausgesprochene Ziel der Stadt Stuttgart.

\subsection{Gesellschaftliche Auswirkungen}

Die Politik steht im Rampenlicht der Öffentlichkeit - und der öffentlichen Kritik. Das Projekt Cuparla bietet den Stadträten und anderen Parlamentariern die Chance, sich an die Spitze der technologischen Innovation zu stellen und damit in dieser Hinsicht zu einem Vorbild für die Bevölkerung zu werden. Die Erfahrungen in Stuttgart zeigen: Innovation in der Politik fördert Innovation durch die Politik.

Die bisherigen Erfahrungen deuten darauf hin, daß innerhalb der Stadtverwaltung sich die Rationalisierungswirkungen und arbeitsplatzschaffenden Wirkungen durch neue Dienstleistungen die Waage halten. Positive Arbeitsplatzwirkungen werden außerhalb der Stadtverwaltung durch die Bereitstellung neuer Dienst- 
leistungen erwartet. Schon jetzt hat das Projekt zu Neueinstellungen bei den beteiligten Projektpartnern sowie zu einer Existenzgründung einer Firma mit mehreren festangestellten Mitarbeitern sowie freien Mitarbeitern geführt. Größere Arbeitsplatzwirkungen wird das Projekt dann entfalten, wenn der Dienst durch die Deutsche Telekom bundesweit auf den Markt gebracht wird und eine Dienstinfrastruktur für viele Gemeinden aufbaut. Da es diese Dienste bisher noch nicht gibt, halten wir es für möglich, daß die Arbeitsplatzwirkung insgesamt positiv ist.

\section{Literaturverzeichnis}

Benford, S./Brown, C.; Reynard, G.; Greenhalgh, C. (1996): Shared spaces: transportation, artificiality, and apaciality. In: Proceedings of the CSCW "96, ACM Press 1996, S. 77-86.

Gryczan, G./Züllighoven, H. (1992): Objektorientierte Systementwicklung - Leitbild und Entwicklungsdokumente. In: Informatik Spektrum, Vol. 15, Nr. 5 Oktober (1992), S. 264-272.

Harrison, S./Dourish, P. (1996): Re-Place-ing Space: The roles of place and space in Collaborative Systems. In: Proceedings of the CSCW "96, ACMPress, New York 1996, S.67-75.

Henderson, D./Card, S. (1986): Rooms: The use of multiple virtual workspaces to reduce space contention in a window-based graphical user interface. In: ACM Transactions on Graphics, Vol. 5, Nr. 3 (Juli 1986), S. 211-243.

Lewe, H. (1995): Computer Aided Team und Produktivität - Einsatzmöglichkeiten und Nutzenpotentiale. Gabler, Wiesbaden 1995.

Pankoke-Babatz, U./Syri, A. (1996): Gemeinsame Arbeitsbereiche: Eine neue Form der Telekooperation?. In: Krcmar/H.; Lewe/H.; Schwabe, G.: Herausforderung Telekooperation - Proceedings der DCSCW 96, Springer, Heidelberg u.a. 1996, S. 51-68.

Reichwald, R./Möslein, K./Sachenbacher, H./Englberger, H./Oldenburg, S. (1998): Telekooperation - Verteilte Arbeits- und Organisationsformen, Springer, Heidelberg u.a. 1998a.

Schwabe, G. (1995): Objekte der Gruppenarbeit - ein Konzept für das Computer Aided Team. Gabler, Wiesbaden 1995.

Schwabe, G. (1998): Pilotierung von Telekooperation. Habilitationsschrift am Lehrstuhl für Wirtschaftsinformatik der Universität Hohenheim, Stuttgart 1998.

Schwabe, G./Krcmar, H. (1996): Der Needs Driven Approach - Eine Methode zur Gestaltung von Telekooperation. In: Krcmar, H./Lewe, H./Schwabe, 
G.: Herausforderung Telekooperation - Proceedings der DCSCW 96, Springer, Heidelberg u.a. 1996.

Schwabe, G./Krcmar, H. (1998): Wettbewerb als Einführungsstrategie von Telekooperation für Entscheidungsträger - Erfahrungen aus dem Projekt Cuparla. In: Wirtschaftsinformatik, Vol. 40 Nr. 3. 1998, S. 200-204.

Vöhringer, B. (1996): Gemeinderatsarbeit heute und Erwartungen an die Unterstützung durch den Computer. Diplomarbeit am Lehrstuhl für Wirtschaftsinformatik der Universität Hohenheim, Stuttgart 1996.

Weiß, D./Krcmar, H. (1996): Workflow-Management: Herkunft und Klassifikation. In: Wirtschaftsinformatik Vol. 38 Nr. 5 (Oktober 1996), S. 503514. 